\title{
ON THE SEMIPRIMITIVITY OF CYCLIC CODES
}

\author{
YVES AUBRY AND PHILIPPE LANGEVIN
}

\begin{abstract}
We prove, without assuming the Generalized Riemann Hypothesis, but with at most one exception, that an irreducible cyclic code $c(p, m, v)$ with $v$ prime and $p$ of index 2 modulo $v$ is a two-weight code if and only if it is a semiprimitive code or it is one of the six sporadic known codes. The result is proved without any exception for index-two irreducible cyclic $c(p, m, v)$ codes with $v$ prime not congruent to 3 modulo 8 . Finally, we prove that these two results hold true in fact for irreducible cyclic code $c(p, m, v)$ such that there is three $p$-cyclotomic cosets modulo $v$.
\end{abstract}

\section{INTRODUCTION}

Irreducible cyclic codes are extensively studied in the literature. They can be defined by three parameters $p, m$ and $v$ and are denoted $c(p, m, v)$ (see section 2 for a precise definition). Such codes with only few different (Hamming) weights are highly appreciated, especially those with exactly two non-zero weights, called two-weight codes. The classification of two-weight codes is a classical problem in coding theory (see [3]); it is still an open problem but recent progress has been made. An infinite family, namely the semiprimitive codes (i.e. when -1 is a power of $p$ modulo $v$ ), and eleven sporadic examples are known. Schmidt and White in [9] provided evidence to conjecture that this is the whole story:

Conjecture 1. An irreducible cyclic code $c(p, m, v)$ is a two-weight code if and only if it is a semiprimitive code or it is one of the eleven sporadic known codes.

They proved their conjecture, conditional on the Generalized Riemann Hypothesis (G.R.H.), for index-two codes, that is when $p$ has index 2 modulo $v$. Note that semiprimitive codes have two non-zero weights and thus only the "only if" part had to be proved.

We considered in [1] the conjecture in the binary case and we proved it in a particular case without assuming G.R.H.. Our main result here is a proof of this conjecture without assuming G.R.H. but with at most one exception in the case where $p$ has index 2 and $v$ is prime. We prove before, using near-primitive root densities and conditionally on G.R.H., that for any prime number $p$ there are infinitely many such codes namely index-two irreducible cyclic codes $c(p, m, v)$ with $v$ prime. 
We prove the conjecture without any exception (and without assuming G.R.H.) in the case where $p$ has index 2 and $v$ is a prime not congruent to 3 modulo 8 . Finally, we remark that the results hold true in fact for irreducible cyclic codes $c(p, m, v)$ with $v$ an integer such that there is three $p$-cyclotomic cosets modulo $v$.

\section{IrReducible CyCliC CODES AND MCElieCe Weight-FORMula}

Let us introduce irreducible cyclic codes over a prime finite field (indeed, it is enough for our purpose, namely the classification of twoweight irreducible cyclic codes, to consider such codes over prime fields, as remarked in [9]).

Let $p$ be a prime number and consider the finite field $K$ with $p$ elements. Let $L$ be the extension of degree $m$ of $K$, consider a divisor $n$ of $p^{m}-1$ and write $v=\left(p^{m}-1\right) / n$ (thus $v$ and $p$ are coprime). Let $\zeta$ be a primitive $n$-th root of unity in $L$ (i.e. $\zeta$ is a generator of the cyclic subgroup of order $n$ of the multiplicative group $\left.L^{*}\right)$. We define the $c(p, m, v)$ code to be the image of the following map $\Phi_{m}$ :

$$
\begin{aligned}
\Phi_{m}: \quad L & \longrightarrow K^{n} \\
t & \longmapsto\left(\operatorname{Tr}_{L / K}\left(t \zeta^{-i}\right)\right)_{i=0}^{n-1}
\end{aligned}
$$

where $\operatorname{Tr}_{L / K}$ is the trace of the field $L$ over $K$.

It is a code of length $n$ and dimension $\operatorname{ord}_{n}(p)$, the multiplicative order of $p$ modulo $n$. Every irreducible cyclic code over $K$ can be viewed as a $c(p, m, v)$ code (see [9]), so we can take $c(p, m, v)$ as the definition of irreducible cyclic codes over $K$ of length $n$. The $c(p, m, v)$ codes are known to be projective or saturated according to whether $\operatorname{gcd}(n, p-1)=1$ or $\operatorname{gcd}(n, p-1)=p-1$. As remarked in [9], we may assume the saturated situation.

Now we are interested in the weight $w(t)$ of a codeword $\Phi_{m}(t)$ of such a code, for $t \in L^{*}$. Let $\chi$ be a character of the multiplicative group $L^{*}$ and let

$$
\tau_{L}(\chi)=-\sum_{x \in L^{*}} \chi(x) e^{\frac{2 i \pi}{p} \operatorname{Tr}_{L / K}(x)}
$$

be the Gauss sum associated with $\chi$.

Let $V$ be the subgroup of $L^{*}$ of index $v$ and let $\Gamma$ be the subgroup of characters of $L^{*}$ which are trivial both on $V$ and $K^{*}$. Note that the order of $\Gamma$ is equal to $v \operatorname{gcd}(n, p-1) /(p-1)$ which is just equal to $v$ in the saturated situation. We have the following McEliece formula:

Proposition 2. For any $t \in L^{*}$, the weight $w(t)$ of the codeword $\Phi_{m}(t)$ is given by:

$$
w(t)=\frac{p-1}{p v}\left(p^{m}+\sum_{\chi \in \Gamma \backslash\{1\}} \tau_{L}(\chi) \bar{\chi}(t)\right) .
$$

And, conversely by Fourier inversion 


$$
\tau_{L}(\chi)=\frac{p}{p-1} \sum_{t \in L^{*} / V} w(t) \chi(t) .
$$

One says that $p$ is semiprimitive modulo $v$ when -1 is in the group generated by $p$ in $(\mathbf{Z} / v \mathbf{Z})^{*}$, i.e. when $\operatorname{ord}_{v}(p)$ is even. Note that in this case all the Gauss sums are rational and a $c(p, m, v)$ code is a two-weight code. In the paper we investigate the reciprocal: besides some sporadic known examples, is any two-weight irreducible cyclic code semiprimitive?

\section{THE CASE $v$ SMALL}

Before going further let us treat the case where $v$ is small, i.e. $v=2$ or 3 . We know that a $c(p, m, 2)$ code is a two-weight code, and that the weights can be expressed in term of quadratic Gauss sum (see [7]). In the same way, the weights of a $c(p, m, 3)$ code can be expressed by means of cubic Gauss sums. However, it is hard to give the exact values of the cubic Gauss sums (see [6]), and thus also the weights of such a code. Nevertheless, we have the following characterization:

Proposition 3. A $c(p, m, 3)$ code has two weights if and only if it is semiprimitive (that is here, if and only if $p \equiv 2 \bmod 3$ ).

Proof. Let $\chi$ be a multiplicative character of $L$ of order 3. The number of weights of a $c(p, m, 3)$ code is equal to the number of distincts values taken by the mapping:

$$
L^{*} \ni t \mapsto f(t)=\tau_{L}(\chi) \chi(t)+\tau_{L}(\bar{\chi}) \bar{\chi}(t) .
$$

Let $1 \neq j$ be a cubic root of unity. Let $t$ be such that $\chi(t)=j$. It is easy to see that $f(1)=f(t)$ implies $\tau_{L}(\bar{\chi})=j \tau_{L}(\chi)$, that $f(t)=f\left(t^{2}\right)$ implies $\tau_{L}(\bar{\chi})=\tau_{L}(\chi)$ and that $f(1)=f\left(t^{2}\right)$ implies $\tau_{L}(\bar{\chi})=j^{2} \tau_{L}(\chi)$. Therefore, the code has two weights if and only if there exists a cubic root of unity $\omega$ such that

$$
\tau_{L}(\bar{\chi})=\omega \tau_{L}(\chi)
$$

In particular, since $\tau_{L}(\chi)^{3}$ is an algebraic integer of degree 2 and norm $p^{3 m}$, we deduce that $\tau_{L}(\chi)^{6}=\tau_{L}(\bar{\chi})^{6}=p^{6 m}$. Hence the Gauss sums $\tau_{L}(\chi)$ are pure Gauss sums (see [7] for a definition of a pure Gauss sum). It follows by a theorem of Baumert, Mills and Ward (see Theorem 11.6.4 of [7] for example) that $p$ is semiprimitive modulo 3 .

\section{Infinitely MANy INDEX-TWo $c(p, m, v)$ CODES WITH $v$ PRIME}

For the study of $c(p, m, v)$ codes with $v$ prime and $p$ of index two modulo $v$, we are interested in primitive and near-primitive root densities. 
In 1927, Emil Artin made the following conjecture (called now the Artin's primitive root conjecture): for any integer $\alpha \neq \pm 1$ not a square, the natural density

$$
\lim _{x \rightarrow+\infty} \frac{\sharp\left\{v \text { prime } \mid v \leq x \text { and } \alpha \text { generates } \mathbf{F}_{v}^{*}\right\}}{\sharp\{v \text { prime } \mid v \leq x\}}
$$

exists and is positive. In 1967, Hooley proved this conjecture under the assumption of G.R.H.. In particular, he proved that if $\alpha$ is neither \pm 1 nor a perfect square, then there are infinitely many primes $v$ for which $\alpha$ is a primitive root modulo $v$.

If we ask $\alpha$ to generate only the squares of $\mathbf{F}_{v}^{*}$ and not the whole group $\mathbf{F}_{v}^{*}$, i.e. to have index 2 and not index 1 modulo $v$, we come to the notion of near-primitive roots. Precisely, fix $\alpha \neq \pm 1$ not a perfect power and let $v$ be a prime and $t$ be an integer such that $v \equiv 1(\bmod t)$. Consider

$$
N_{\alpha, t}(x)=\sharp\left\{v \text { prime } \mid v \leq x \text { and } v \not \alpha \text { and } \operatorname{ind}_{v}(\alpha)=t\right\} .
$$

Notice that for $t=1$ this quantity is just the previous one studied by Artin and Hooley. In 2000, Moree introduced in [8] a weighting function depending on $\alpha$ and $t$ and gave an estimation of $N_{\alpha, t}(x)$ assuming G.R.H.. In particular, for $\alpha=p$ a prime number and $t=2$, he proved that

$$
N_{p, 2}(x)=\sum_{\substack{v \text { odd prime } \\ v \leq x}} \frac{\varphi\left(\frac{v-1}{2}\right)}{v-1}+O\left(\frac{x \log \log x}{\log ^{2} x}\right) .
$$

This implies that there exist infinitely many primes $v$ such that $p$ has index 2 modulo $v$.

In particular, we have:

Proposition 4. Conditionally on G.R.H., for any prime number $p$ there are infinitely many index-two irreducible cyclic codes $c(p, m, v)$ with $v$ prime.

\section{NeCESSARY CONDITIONS ON TWO-WEIGHT CODES}

The irreducible cyclic codes $c(p, m, v)$, with $v$ a prime number and with $p$ of index 2 modulo $v$, range in two families: the first one with $v \equiv 1(\bmod 4)$ and the second one with $v \equiv 3(\bmod 4)$. If $v \equiv 1$ $(\bmod 4)$, then -1 is a square modulo $v$ and since $p$ generates the squares modulo $v$, we are reduced to the semiprimitive case. This lead us to consider the second case, where -1 is not a square modulo $v$. Moreover, in view of Proposition 3, we can suppose that $v$ is greater than 3.

Hence, let us consider a prime number $p$ and an integer $v$ satisfying the following $(\sharp)$ conditions:

(a) $v$ is a prime greater than 3 ,

(b) $\operatorname{ord}_{v}(p)=(v-1) / 2$ i.e. $p$ has index 2 modulo $v$,

(c) $v \equiv 3(\bmod 4)$. 
Let $f$ be the multiplicative order of $p$ modulo $v$. Note that $f$ divides $m$, and we set $s=m / f$. It is shown in [4] that if a $c(p, m, v)$ code with $v$ satisfying the $(\sharp)$ conditions has two weights then:

$$
\frac{v+1}{4}=p^{h s} .
$$

We give, now, a more precise result:

Theorem 5. If a $c(p, m, v)$ code with $v$ satisfying the $(\sharp)$ conditions is a two-weight code then we have:

$$
m=\operatorname{ord}_{v}(p) .
$$

Proof. Since $p$ has index 2 modulo $v$, then $p$ is a square modulo $v$, and

$$
(p)=P P^{\prime}
$$

splits in the extension $\mathbf{Q}(\sqrt{-v}) / \mathbf{Q}$. We have that the norm

$$
N_{\mathbf{Q}(\sqrt{-v}) / \mathbf{Q}}(P)=p
$$

and that $P^{h}=(\alpha)$ is a principal ideal (since $h$ is the ideal class number of $\mathbf{Q}(\sqrt{-v})$ ), with $\alpha=(a+b \sqrt{-v}) / 2$ (with $a, b \in \mathbf{Z}$ ) an algebraic integer of $\mathbf{Q}(\sqrt{-v})$. Taking norms, we obtain $p^{h}=\left(a^{2}+v b^{2}\right) / 4$ and since $a$ and $b$ cannot be zero in this situation, we conclude that

$$
\frac{v+1}{4} \leq p^{h} .
$$

But by $(5)$ a $c(p, m, v)$ code with $v$ satisfying the $(\sharp)$ conditions has two weights if and only if

$$
\frac{v+1}{4}=p^{h s}
$$

Thus, $p^{h s} \leq p^{h}$ and $s=1$.

Then, the previously defined parameter $s$ appearing in [4] and [9] is equal to 1 under the $(\sharp)$ conditions. In particular, we have:

Corollary 6. If a $c(p, m, v)$ code with $v$ satisfying the $(\sharp)$ conditions is a two-weight code then

$$
\frac{v+1}{4}=p^{h} .
$$

where $h$ is the class number of the imaginary quadratic number field $\mathbf{Q}(\sqrt{-v})$. In particular, such a code is completely defined by the parameter $v$.

Furthermore, we have the following necessary condition on $p$ for twoweight $c(p, m, v)$ code with $v$ satisfying the $(\sharp)$ conditions:

Corollary 7. If a $c(p, m, v)$ code with $v$ satisfying the $(\sharp)$ conditions has two weights, then $p$ is the least prime which totally splits in the extension $\mathbf{Q}(\sqrt{-v}) / \mathbf{Q}$, i.e. $p$ is the least prime which is a square modulo $v$. 
Proof. Indeed, if $\ell$ is a prime which totaly splits in $\mathbf{Q}(\sqrt{-v}) / \mathbf{Q}$, then the previous proof implies that $\ell^{h} \geq \frac{v+1}{4}=p^{h}$ which gives $\ell \geq p$.

\section{Main Results}

Using the previous section, we can state the following result which can also be derived from the proof of lemma 4.1. of [4].

Theorem 8. There is no two-weight $c(p, m, v)$ code with $v$ satisfying the $(\sharp)$ conditions and with $v \equiv 7(\bmod 8)$. Hence, Conjecture 1 holds true for index-two irreducible cyclic $c(p, m, v)$ codes with $v$ a prime not congruent to 3 modulo 8.

Proof. Since $v \equiv 7(\bmod 8)$, it follows that 2 is a square modulo $v$, and the ideal (2) splits in the extension $\mathbf{Q}(\sqrt{-v}) / \mathrm{Q}$. By Corollary 7, we conclude that $p=2$. But we proved in [1] that there exists no two-weight binary $c(p, m, v)$ code with $v$ satisfying the $(\sharp)$ conditions, so we get the non-existence of such codes. Hence, this proves the conjecture since the case $v \equiv 1(\bmod 4)$ is trivial, as quoted in the previous section, and the last case $v \equiv 3(\bmod 4)$ is divided in two subcases : when $v \equiv 7(\bmod 8)$, which is now solved, and when $v \equiv 3$ $(\bmod 8)$ which is the remainder case.

Actually, we will consider now a more general approach using the identity of Corollary 6 but with at most one exception.

If a $c(p, m, v)$ code with $v$ satisfying the $(\sharp)$ conditions has two weights then we have the following relation

$$
\frac{v+1}{4}=p^{h},
$$

where $h$ is the class number of the imaginary quadratic number field $\mathbf{Q}(\sqrt{-v})$ (see Corollary 6).

In 1935, Siegel gave a non-effective lower bound on the residue at $s=$ 1 of the L-function $L\left(s, \chi_{v}\right)$ associated to the primitive odd Dirichlet character $\chi_{v}$ of $\mathbf{Q}(\sqrt{-v})$. Tatuzawa, in 1951, proved an effective lower bound of $L\left(1, \chi_{v}\right)$ but with at most one exception (see [10] and see also [5] for a simple proof): if $0<\varepsilon<1 / 2$ and $v \geq \max \left(e^{1 / \varepsilon}, e^{11.2}\right)$, then

$$
L\left(1, \chi_{v}\right) \geq 0.655 \varepsilon v^{-\varepsilon} \text {. }
$$

Since the class number $h$ of $\mathbf{Q}(\sqrt{-v})$ with $-v \equiv 1(\bmod 4)$ is linked to $L\left(1, \chi_{v}\right)$ by the following Dirichlet class number formula:

$$
L\left(1, \chi_{v}\right)=\frac{\pi h}{\sqrt{v}},
$$

we can use Tatuzawa theorem to get an upper bound on $v$.

Proposition 9. There exists at most one two-weight $c(p, m, v)$ code with $v \geq 10^{8}$ satisfying the $(\sharp)$ conditions. 
TABLE 1 . Sporadic $c(p, m, v)$ codes with $v$ satisfying the $(\sharp)$ conditions and $v \leq 10^{8}$.

\begin{tabular}{|c|cccccc|}
\hline$v$ & 11 & 19 & 67 & 107 & 163 & 499 \\
\hline$p$ & 3 & 5 & 17 & 3 & 41 & 5 \\
\hline$h$ & 1 & 1 & 1 & 3 & 1 & 3 \\
\hline
\end{tabular}

Proof. Let $\varepsilon=1 / \log \left(10^{8}\right) \in(0,1 / 2)$. For $v \geq \max \left(e^{1 / \varepsilon}, e^{11.2}\right)=10^{8}$, we have, with at most one exception:

$$
L\left(1, \chi_{v}\right) \geq 0.655 \varepsilon v^{-\varepsilon}=0.035 v^{-0.054} .
$$

Now, $\frac{v+1}{4}=p^{h} \geq 2^{h}$ implies that $\log \frac{v+1}{4} \geq h \log 2$. By the Dirichlet class number formula, we get:

$$
\log \frac{v+1}{4} \geq \frac{\sqrt{v} L\left(1, \chi_{v}\right)}{\pi} \log 2
$$

But, for $v \geq 10^{8}$, we have on one hand $\log \frac{v+1}{4} \geq 17.03$ and on the other hand $\frac{\sqrt{v} L\left(1, \chi_{v}\right)}{\pi} \log 2>28.55$ by Tatuzawa theorem. Thus, there exists no $v \geq 10^{8}$ such that $\frac{v+1}{4}=p^{h}$, with at most one exception.

Now, we make an exhaustive research of the primes $v \leq 10^{8}$ such that $(v+1) / 4$ is a power of a prime $p$. Then, for such primes $v$, we check whether $(v+1) / 4=p^{h(v)}$ holds true or not, with $h(v)$ the class number of $\mathbf{Q}(\sqrt{-v})$. Actually, we recover the following sporadic known examples of Table 1.

Thus, we have proved the following theorem:

Theorem 10. Any two-weight irreducible cyclic $c(p, m, v)$ code where $p$ has index two modulo a prime $v$ and which is not one of the six sporadic examples of Table 1 is semiprimitive, with at most one exception. Hence, Conjecture 1 is true, with at most one exception, for all index-two $c(p, m, v)$ codes with $v$ prime.

\section{Cyclotomic cosets}

Let $p$ be a prime. For any integer $v$ prime to $p$, consider on the ring $\mathbf{Z} / v \mathbf{Z}$ the equivalence relation given by: for $a, b \in \mathbf{Z} / v \mathbf{Z}$, we set $a \sim b$ if and only if there exists $t \in \mathbf{Z}$ such that $a=b p^{t}$. The equivalence classes for this equivalence relation are the so-called $p$-cyclotomic cosets modulo $v$.

Recall that the order $\operatorname{ord}_{v}(g)$ of an element $g$ of the multiplicative group $(\mathbf{Z} / v \mathbf{Z})^{*}$ divides the order $\varphi(v)$ of this group, where $\varphi$ is the Euler function. We denote by $\operatorname{ind}_{v}(g)$ the index of $g$ modulo $v$ i.e.

$$
\operatorname{ind}_{v}(g)=\frac{\varphi(v)}{\operatorname{ord}_{v}(g)} .
$$

Then $\operatorname{ind}_{v}(g)=\left[(\mathbf{Z} / v \mathbf{Z})^{*}:\langle g\rangle\right]$ where $\langle g\rangle$ denotes the subgroup of $(\mathbf{Z} / v \mathbf{Z})^{*}$ generated by $g$. But the number $\gamma(p, v)$ of $p$-cyclotomic cosets 
modulo $v$ is also equal to the number of irreducibles polynomials in the decomposition of the polynomial $X^{v}-1$ over $\mathbf{F}_{p}$, thus it is equal to

$$
\gamma(p, v)=\sum_{d \mid v} \frac{\varphi(d)}{\operatorname{ord}_{d}(p)}=\sum_{d \mid v} \operatorname{ind}_{d}(p)
$$

with the convention that $\operatorname{ind}_{1}(p)=1$. For example, the condition $\gamma(p, v)=2$ is equivalent to $\operatorname{ind}_{v}(p)=1$, that is $p$ is a primitive root modulo $v$.

Proposition 11. Let $v$ be an integer. The ring $\mathbf{Z} / v \mathbf{Z}$ contains exactly 3 p-cyclotomic cosets if and only if one of the following holds:

(i) $v$ is a prime and $p$ has index $2 \bmod v$;

(ii) $v$ is the square of a prime and $p$ has index $1 \bmod v$.

Proof. By (8) we have $\gamma(p, v)=3$ if and only if $\operatorname{ind}_{v}(p)=2$ and $v$ has no proper divisor, or $\operatorname{ind}_{v}(p)=1$ and $v$ has a unique proper divisor. The proposition is then proved.

Proposition 12. Let $v$ be an integer. If the ring $\mathbf{Z} / v \mathbf{Z}$ contains exactly three $p$-cyclotomic cosets then any $c(p, m, v)$ code has at most three nonzero weights.

Proof. The result is in fact much general: the number of weights is less or equal than the number of cyclotomic cosets. It follows from the fact that the weight of a codeword of a $c(p, m, v)$ code is invariant under $t \mapsto t \zeta$ and under $t \mapsto t^{p}$; see Theorem 2.5 of [2] for a detailed proof.

The case $(i i)$ of Proposition 11 falls into the semiprimitive case since $p$ generates the whole group $(\mathbf{Z} / v \mathbf{Z})^{*}$ and thus contains -1 .

Finally, we have proved the following result:

Theorem 13. If $v$ is an integer such that there is three $p$-cyclotomic cosets modulo $v$ then any two-weight irreducible cyclic code $c(p, m, v)$ which is not one of the six sporadic examples of Table 1 is semiprimitive, with at most one exception. Hence, Conjecture 1 holds true, with at most one exception, for all $c(p, m, v)$ codes with $v$ an integer such that there is three p-cyclotomic cosets modulo $v$.

Proof. If a binary irreducible cyclic code with three-cyclotomic cosets has two weights then it is semiprimitive. Indeed, by Proposition 11, an irreducible cyclic code with three-cyclotomic cosets leads to two cases. The first one leads $c(p, m, v)$ codes with $v$ a square of a prime and $p$ of index 1 modulo $v$ which gives a semiprimitive code.

The other case leads to $c(p, m, v)$ codes with $v$ a prime and $p$ of index 2 modulo $v$ (the so-called index-two codes). When $v \equiv 1(\bmod 4)$, we saw that we obtain a semiprimitive code. When $v \equiv 3(\bmod 4)$, we obtain $c(p, m, v)$ codes with $v$ satisfying the $(\sharp)$ conditions. In the case 
where $p=2$, i.e. the binary case, we found in [1] that there is no two-weight codes. When $p \neq 2$, theorem 10 gives the result.

\section{REFERENCES}

[1] Y. Aubry and P. Langevin, On the weights of binary irreducible cyclic codes, Coding and Cryptography, Springer Lecture Notes in Computer Science, vol. 3969, 46-54 (2006).

[2] R. W. Fitzgerald and J. L. Yucas, Sums of Gauss sums and weights of irreducible codes, Finite Field and Their Applications 11 (2005), 89-110.

[3] R. Calderbank, W. M. Kantor, The geometry of two-weight codes, Technical report, Bell Laboratory, 1978.

[4] Ph. Langevin, A new class of two weight codes, Finite fields and their applications, Glasgow 1995, London Math. Soc. Lecture Note Ser. 233, 181-187 (1996).

[5] S. Louboutin, Simple proofs of the Siegel-Tatuzawa and Brauer-Siegel theorems, Colloq. Math. 108 (2007), no. 2, 277-283.

[6] C. R. Matthews, Gauss sums and elliptic functions I. The Kummer sums, Invent. Math., 52 (1979), 163-185.

[7] B. C. Berndt, R. J. Evans and K. S. Williams, Gauss and Jacobi Sums, WileyInterscience, N. Y., 1998.

[8] P. Moree, Asymptotically exact heuristics for (near) primitive roots, J. Number Theory 83 (2000), no. 1, 155-181.

[9] B. Schmidt and C. White, All two-weight irreducible cyclic codes ?, Finite Fields and Their Applications 8 (2002), 1-17.

[10] T. Tatuzawa, On a theorem of Siegel, Jap. J. Math. 21 (1951), 163-178 (1952).

Institut de Mathématiques de Toulon, Université Du Sud ToulonVAR, FRANCE.

E-mail address: $\{$ yaubry, langevin\}@univ-tln.fr 